\title{
Apixaban versus Enoxaparin for Thromboprophylaxis in Medically Ill Patients
}

\author{
Samuel Z. Goldhaber, M.D., Alain Leizorovicz, M.D., Ajay K. Kakkar, M.D., Ph.D., \\ Sylvia K. Haas, M.D., Ph.D., Geno Merli, M.D., Robert M. Knabb, Ph.D., \\ and Jeffrey I. Weitz, M.D., for the ADOPT Trial Investigators*
}

\section{A BSTRACT}

\section{BACKGROUND}

The efficacy and safety of prolonging prophylaxis for venous thromboembolism in medically ill patients beyond hospital discharge remain uncertain. We hypothesized that extended prophylaxis with apixaban would be safe and more effective than short-term prophylaxis with enoxaparin.

\section{METHODS}

In this double-blind, double-dummy, placebo-controlled trial, we randomly assigned acutely ill patients who had congestive heart failure or respiratory failure or other medical disorders and at least one additional risk factor for venous thromboembolism and who were hospitalized with an expected stay of at least 3 days to receive apixaban, administered orally at a dose of $2.5 \mathrm{mg}$ twice daily for 30 days, or enoxaparin, administered subcutaneously at a dose of $40 \mathrm{mg}$ once daily for 6 to 14 days. The primary efficacy outcome was the 30-day composite of death related to venous thromboembolism, pulmonary embolism, symptomatic deep-vein thrombosis, or asymptomatic proximal-leg deep-vein thrombosis, as detected with the use of systematic bilateral compression ultrasonography on day 30 . The primary safety outcome was bleeding. All efficacy and safety outcomes were independently adjudicated.

\section{RESULTS}

A total of 6528 subjects underwent randomization, 4495 of whom could be evaluated for the primary efficacy outcome - 2211 in the apixaban group and 2284 in the enoxaparin group. Among the patients who could be evaluated, $2.71 \%$ in the apixaban group (60 patients) and 3.06\% in the enoxaparin group (70 patients) met the criteria for the primary efficacy outcome (relative risk with apixaban, 0.87 ; $95 \%$ confidence interval [CI], 0.62 to $1.23 ; \mathrm{P}=0.44$ ). By day 30 , major bleeding had occurred in $0.47 \%$ of the patients in the apixaban group (15 of 3184 patients) and in $0.19 \%$ of the patients in the enoxaparin group (6 of 3217 patients) (relative risk, 2.58; 95\% CI, 1.02 to $7.24 ; \mathrm{P}=0.04)$.

\section{CONCLUSIONS}

In medically ill patients, an extended course of thromboprophylaxis with apixaban was not superior to a shorter course with enoxaparin. Apixaban was associated with significantly more major bleeding events than was enoxaparin. (Funded by BristolMyers Squibb and Pfizer; ClinicalTrials.gov number, NCT00457002.)
From the Cardiovascular Division, Department of Medicine, Brigham and Women's Hospital and Harvard Medical School, Boston (S.Z.G.); Unité de Pharmacologie Clinique, Université Claude Bernard Lyon I, Lyon, France (A.L.); Thrombosis Research Institute and University College London — both in London (A.K.K.); Institute for Experimental Oncology and Therapy Research, Technical University of Munich, Munich, Germany (S.K.H.); Jefferson Medical College, Thomas Jefferson University Hospitals, Philadelphia (G.M.); BristolMyers Squibb, Princeton, NJ (R.M.K.); and the Thrombosis and Atherosclerosis Research Institute and McMaster University — both in Hamilton, ON, Canada (J.I.W.). Address reprint requests to $\mathrm{Dr}$. Goldhaber at Brigham and Women's Hospital, Cardiovascular Division, 75 Francis St., Boston, MA 02115, or at sgoldhaber@partners.org.

*A complete list of the members and investigators in the Apixaban Dosing to Optimize Protection from Thrombosis (ADOPT) trial is provided in the Supplementary Appendix, available at NEJM.org.

This article (10.1056/NEJMoal110899) was published on November 13, 2011, and updated on November 17, 2011, at NEJM.org.

N Engl J Med 2011;365:2167-77.

Copyright (๑) 2011 Massachusetts Medical Society. 
ENOUS THROMBOEMBOLISM IS A COMmon and potentially fatal complication in hospitalized surgical patients and acutely ill medical patients. ${ }^{1}$ The benefits of providing pharmacologic thromboprophylaxis over the entire course of the hospital stay have been validated, with efficacy and safety shown in both populations. ${ }^{2-5}$

Among high-risk surgical patients, such as those undergoing total hip replacement, extended thromboprophylaxis in the period after hospital discharge has reduced the rate of both asymptomatic and symptomatic venous thromboembolism. ${ }^{6}$ On the basis of these findings, current practice guidelines recommend extended thromboprophylaxis in such patients. ${ }^{7}$

One study (the Extended Prophylaxis for Venous Thromboembolism in Acutely Ill Medical Patients with Prolonged Immobilization trial [EXCLAIM; ClinicalTrials.gov number, NCT00077753]) ${ }^{8}$ evaluated the potential advantage of extending pharmacologic prophylaxis with enoxaparin beyond the period of hospitalization in acutely ill medical patients. Although the rates of venous thromboembolism were lower with extended thromboprophylaxis, this benefit was offset by a significant increase in major bleeding. In the MAGELLAN study (Venous Thromboembolic Event [VTE] Prophylaxis in Medically Ill Patients, NCT00571649), ${ }^{9}$ extended prophylaxis with rivaroxaban was compared with short-term prophylaxis with enoxaparin followed by placebo. This trial also showed lower rates of venous thromboembolism with extended thromboprophylaxis, but there were more major bleeding events with rivaroxaban than with enoxaparin.

Apixaban is an orally active direct inhibitor of activated factor $\mathrm{X}$ (factor $\mathrm{Xa}$ ) with established efficacy and safety for the prevention of venous thromboembolism after elective hip or knee replacement ${ }^{10,11}$ and for the prevention of stroke in patients with atrial fibrillation. ${ }^{12,13}$ In the Apixaban Dosing to Optimize Protection from Thrombosis (ADOPT) trial, we evaluated the potential of apixaban to prevent venous thromboembolism in acutely ill medical patients during hospitalization and in the extended period after their discharge from the hospital.

\section{METHODS}

STUDY OVERSIGHT

The trial was designed and led by an executive committee that included academic investigators and by representatives of the sponsors (BristolMyers Squibb and Pfizer). The study protocol, including the statistical analysis plan, is available with the full text of this article at NEJM.org. The study was conducted according to the ethical principles stated in the Declaration of Helsinki. Approval was obtained from the appropriate ethics committee at each site, and all patients provided written informed consent. All the authors participated in the design of the trial and the planning of the analyses. The first author wrote the first draft of the manuscript with input from all the coauthors and revised the subsequent drafts on the basis of input from the coauthors. All the authors vouch for the accuracy and completeness of the reported data and for the fidelity of the study to the protocol.

\section{PATIENTS}

Male and female patients, 40 years of age or older, were considered for participation in the study if they were hospitalized for congestive heart failure, acute respiratory failure, infection (without septic shock), acute rheumatic disorder, or inflammatory bowel disease and had an expected hospital stay of at least 3 days. Except for patients with congestive heart failure or respiratory failure, eligible patients had to have at least one of the following additional risk factors: an age of 75 years or older, previous documented venous thromboembolism or a history of venous thromboembolism for which they received anticoagulation for at least 6 weeks, cancer, a body-mass index (the weight in kilograms divided by the square of the height in meters) of 30 or more, receipt of estrogenic hormone therapy, or chronic heart failure or respiratory failure. In addition, all patients had to be moderately or severely restricted in their mobility. Moderately restricted mobility allowed for walking within the hospital room or to the bathroom. Severely restricted mobility was defined as being confined to bed or to a chair at the bedside.

Patients were excluded if they had confirmed venous thromboembolism; a disease requiring ongoing treatment with a parenteral or oral anticoagulant agent; active liver disease, anemia or thrombocytopenia; severe renal disease (creatinine clearance of $<30 \mathrm{ml}$ per minute as estimated by the method of Cockcroft and Gault); a known or suspected allergy to enoxaparin; or prior heparin-induced thrombocytopenia or if they were taking two or more antiplatelet agents or aspirin at a dose higher than $165 \mathrm{mg}$ per day. 
Patients were also excluded if they had undergone a surgical procedure in the previous 30 days that might be associated with a risk of bleeding, had received anticoagulant prophylaxis for venous thromboembolism in the previous 14 days, were actively bleeding or were at high risk for bleeding; or had invasive procedures planned or scheduled during the treatment period. In addition, patients were excluded if they had one of the following abnormal laboratory findings: a hemoglobin level of less than $9 \mathrm{~g}$ per deciliter, a platelet count of less than 100,000 per cubic millimeter, an alanine or aspartate aminotransferase level more than twice the upper limit of the normal range, or direct or total bilirubin levels more than 1.5 times the upper limit of the normal range. Finally, women who might become pregnant, were pregnant, were breast-feeding, or were unwilling or unable to use an acceptable method of contraception were not eligible.

\section{STUDY DESIGN}

This trial was an international, multicenter, randomized, double-blind, controlled study. Patients were randomly assigned, in a 1:1 ratio, to receive apixaban, administered orally at a dose of $2.5 \mathrm{mg}$ twice daily for 30 days, or enoxaparin, administered subcutaneously at a dose of $40 \mathrm{mg}$ once daily during their stay in the hospital, for a minimum of 6 days. Randomization was performed through a central telephone system with the use of a computer-generated randomization list. The maximum interval allowed between admission to the hospital and randomization was 72 hours. The day of randomization was defined as day 1 of the study. In-person follow-up visits were scheduled on study days $30 \pm 2$ and $90 \pm 7$, with telephone contact at days 14 and 60. A systematic compression ultrasound examination was to be performed at the time of discharge (but no earlier than day 5 and no later than day 14) and at day 30 . All compression ultrasound examinations were recorded for submission to an independent central adjudication committee whose members were unaware of the treatment assignments.

\section{TREATMENTS}

The study medications were packaged in identical-appearing dispensing kits. Patients who were randomly assigned to apixaban received daily injections of an enoxaparin placebo for a minimum of 6 days. After 6 days, the decision to discontinue the parenteral study drug was made at the discretion of the investigators. Patients who were randomly assigned to enoxaparin received tablets containing an apixaban placebo for 30 days. Concomitant treatment with aspirin at doses above $165 \mathrm{mg}$ per day was prohibited.

\section{OUTCOME MEASURES}

The primary efficacy outcome was a composite during the 30-day treatment period of death related to venous thromboembolism (i.e., sudden death for which pulmonary embolism could not be excluded as a cause), fatal or nonfatal pulmonary embolism, symptomatic deep-vein thrombosis, or asymptomatic proximal-leg deep-vein thrombosis as detected with the use of systematic bilateral compression ultrasonography. All components of the primary efficacy outcome were adjudicated by the independent central adjudication committee.

A key secondary efficacy outcome was the composite of total venous thromboembolism and death related to venous thromboembolism occurring from the time of randomization to the time the blinded parenteral therapy was discontinued (the parenteral-treatment period). Additional secondary efficacy outcomes included symptomatic deep-vein thrombosis or nonfatal pulmonary embolism occurring during the 60-day followup period, death from any cause occurring during the 30-day treatment period, and death from any cause occurring during the entire 90-day study period (i.e., the treatment period plus the follow-up period).

The main safety outcomes included major bleeding, clinically relevant nonmajor bleeding, and all bleeding reported by investigators; myocardial infarction; stroke; thrombocytopenia; and death from any cause. Each of these events was reviewed and adjudicated by the independent central adjudication committee. Bleeding was categorized as major if it was fatal or overt and was accompanied by one or more of the following: a decrease in hemoglobin of $2 \mathrm{~g}$ or more per deciliter over a 24-hour period; transfusion of 2 or more units of packed red cells; or intracranial, intraspinal, intraocular, pericardial, or retroperitoneal bleeding, bleeding that occurred in an operated joint that required reoperation or intervention, or intramuscular bleeding with the compartment syndrome. Clinically relevant nonmajor bleeding was defined as acute, clinically overt bleeding that did not meet the criteria for classification as a major bleeding event but did meet 
at least one of the following criteria: epistaxis that required medical attention or persisted for 5 minutes or more, gastrointestinal bleeding containing frank blood or coffee-ground material that tested positive for blood, endoscopically confirmed bleeding, spontaneous hematuria or hematuria persisting for 24 hours or more after urinary-tract catheterization, unusual bruising, radiographically confirmed hematoma, or hemoptysis.

\section{STATISTICAL ANALYSIS}

We estimated that with a sample of 6524 patients randomly assigned in a 1:1 ratio to receive apixaban or enoxaparin, the study would have $90 \%$ power to show the superiority of apixaban with respect to the primary efficacy outcome, at a one-sided alpha level of 0.025 , assuming a true event rate of $2.5 \%$ in the apixaban group and $4.0 \%$ in the enoxaparin group. For the noninferiority test of the first secondary outcome (the composite of total venous thromboembolism and death related to venous thromboembolism during the parenteral-treatment period), the study would have $85 \%$ power to show the noninferiority of apixaban, at a one-sided alpha level of 0.025 , assuming a true event rate of $1.6 \%$ in the apixaban group and $2.0 \%$ in the enoxaparin group, with a noninferiority margin of 1.43 for the relative risk. These estimates were based on the use of the Mantel-Haenszel test for superiority and the test for noninferiority of Yanagawa, Tango, and Hiejima, stratified according to history or no history of venous thromboembolism and status with respect to cancer, and on the assumption that $10 \%$ of the compression ultrasound examinations would not be able to be evaluated.

The primary efficacy data set consisted of all randomly assigned patients in whom compression ultrasonography was performed at any time up to the last scheduled visit during the treatment period, with results that could be evaluated and were adjudicated; patients who had an event of venous thromboembolism, confirmed by adjudication, during the treatment period at any time from randomization to the last scheduled visit during the treatment period; or patients who died from a cause related to venous thromboembolism during the treatment period. To control the overall type I error rate, a sequential test procedure was planned to compare the effect of apixaban with that of enoxaparin with respect to the primary efficacy outcome at day 30 and with respect to the first secondary outcome at the end of the parenteral-treatment period.
The superiority of apixaban with respect to the primary outcome was first tested with the use of the Mantel-Haenszel test, stratified according to history or no history of venous thromboembolism and status with respect to cancer. If significant superiority were established for apixaban with respect to the primary outcome, a noninferiority test would then be performed on the first secondary outcome, with the use of the test for noninferiority of Yanagawa, Tango, and Hiejima, stratified according to history or no history of venous thromboembolism and status with respect to cancer.

The analysis of safety end points was performed on data from the treated population, which consisted of all patients who received at least one dose of a study drug. Adjudicated major bleeding events were summarized according to onset during the treatment period and onset during the follow-up period. An independent data and safety monitoring board reviewed the incidences of major bleeding and events of venous thromboembolism on an ongoing basis, primarily to assess safety. A formal interim efficacy analysis was performed after $50 \%$ of the planned patients had been enrolled.

\section{RESULTS}

\section{STUDY PATIENTS}

From June 2007 through February 2011, a total of 6758 acutely ill medical patients were enrolled at 302 centers in 35 countries, and 6528 subjects were randomly assigned to receive short-term prophylaxis with enoxaparin or extended prophylaxis (30 days) with apixaban. In total, 4495 patients could be evaluated for the primary efficacy outcome at day 30 . Figure 1 shows the number of patients who were enrolled, who underwent randomization, and who were included in the safety and efficacy analyses. The demographic characteristics of the study population, the primary diagnoses at enrollment, and additional risk factors are shown in Table 1. Among subjects who received at least one dose of the randomly assigned study medication, the mean $( \pm S D)$ duration of exposure to apixaban was $24.9 \pm 10.0$ days, and the mean exposure to enoxaparin was $7.3 \pm 4.0$ days.

\section{EFFICACY}

The primary efficacy outcome, evaluated at day 30, occurred in $2.71 \%$ of the patients who were randomly assigned to receive extended prophylaxis 


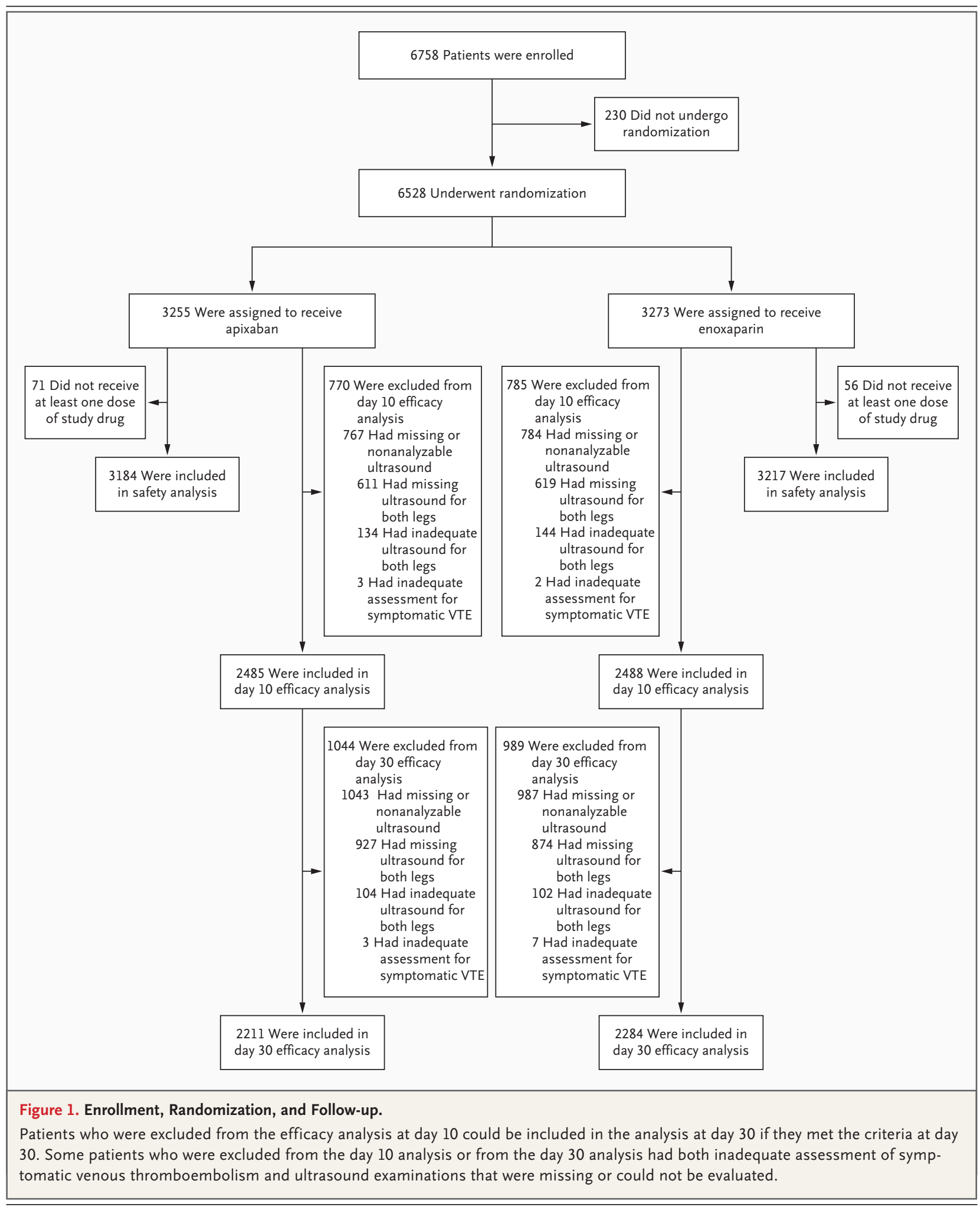

with apixaban (60 of the 2211 patients in the primary efficacy data set) as compared with $3.06 \%$ term prophylaxis with enoxaparin (70 of 2284 paof the patients who were assigned to receive shorttients) (relative risk with apixaban, 0.87 ; 95\% confidence interval [CI], 0.62 to $1.23 ; \mathrm{P}=0.44)$. The key 


\begin{tabular}{|c|c|c|}
\hline Variable & Apixaban $(\mathrm{N}=3255)$ & Enoxaparin $(\mathrm{N}=3273)$ \\
\hline \multicolumn{3}{|l|}{ Demographic characteristics } \\
\hline \multicolumn{3}{|l|}{ Age $-y r$} \\
\hline Mean & $66.8 \pm 12.0$ & $66.7 \pm 12.0$ \\
\hline Median & 68.0 & 67.0 \\
\hline Range & $40-101$ & $40-98$ \\
\hline \multicolumn{3}{|l|}{ Age distribution — no. (\%) } \\
\hline$<65 \mathrm{yr}$ & $1401(43.0)$ & $1411(43.1)$ \\
\hline 65 to $<75 \mathrm{yr}$ & $890(27.3)$ & $884(27.0)$ \\
\hline$\geq 75 \mathrm{yr}$ & $964(29.6)$ & 978 (29.9) \\
\hline \multicolumn{3}{|l|}{ Sex - no. (\%) } \\
\hline Male & $1626(50.0)$ & $1577(48.2)$ \\
\hline Female & $1629(50.0)$ & $1696(51.8)$ \\
\hline \multicolumn{3}{|l|}{ Race or ethnic group — no. (\%) $\dagger$} \\
\hline White & $2474(76.0)$ & $2476(75.6)$ \\
\hline Black & $292(9.0)$ & $304(9.3)$ \\
\hline American Indian or Alaskan Native & $9(0.3)$ & $2(0.1)$ \\
\hline Asian & $320(9.8)$ & $326(10.0)$ \\
\hline Other & $145(4.5)$ & $149(4.6)$ \\
\hline \multicolumn{3}{|l|}{ Reason for hospitalization - no. (\%) } \\
\hline Congestive heart failure & $1270(39.0)$ & $1246(38.1)$ \\
\hline Acute respiratory failure & $1208(37.1)$ & $1213(37.1)$ \\
\hline Infection, without septic shock & $701(21.5)$ & $746(22.8)$ \\
\hline Acute rheumatic disorder & $39(1.2)$ & $36(1.1)$ \\
\hline Inflammatory bowel disease & $26(0.8)$ & $23(0.7)$ \\
\hline Other & $6(0.2)$ & $5(0.2)$ \\
\hline Not reported & $5(0.2)$ & $4(0.1)$ \\
\hline \multicolumn{3}{|l|}{ Additional risk factors — no. (\%) } \\
\hline Previous venous thromboembolism & $141(4.3)$ & $124(3.8)$ \\
\hline History of cancert: & $312(9.6)$ & $320(9.8)$ \\
\hline Active cancer & $113(3.5)$ & $98(3.0)$ \\
\hline Remote cancer & $199(6.1)$ & $222(6.8)$ \\
\hline NYHA Class of chronic heart failure & $1531(47.0)$ & $1537(47.0)$ \\
\hline I & $60(1.8)$ & $47(1.4)$ \\
\hline II & $228(7.0)$ & $240(7.3)$ \\
\hline III & $854(26.2)$ & $833(25.5)$ \\
\hline IV & $380(11.7)$ & $411(12.6)$ \\
\hline Not reported & $9(0.3)$ & $6(0.2)$ \\
\hline Chronic respiratory failure & $1683(51.7)$ & $1702(52.0)$ \\
\hline Body-mass index $\geq 30 \mathbb{\int}$ & $1448(44.5)$ & $1451(44.3)$ \\
\hline Estrogenic hormone therapy & $49(1.5)$ & $27(0.8)$ \\
\hline \multicolumn{3}{|l|}{ Mobility at randomization } \\
\hline Severely restricted & $846(26.0)$ & $929(28.4)$ \\
\hline Moderately restricted & $2388(73.4)$ & $2323(71.0)$ \\
\hline
\end{tabular}

* Plus-minus values are means \pm SD. There were no significant differences between the two treatment groups in any of the baseline characteristics. NYHA denotes New York Heart Association.

$\uparrow$ Race or ethnic group was self-reported. The case-report forms for $0.4 \%$ of the patients in each group did not include information about race.

+ Patients were considered to have active cancer if they had been receiving treatment for cancer within the previous year and were considered to have remote cancer if treatment for cancer ended more than a year before they were enrolled in the study.

$\int$ The body-mass index is the weight in kilograms divided by the square of the height in meters.

9 Moderately restricted mobility was defined as the ability to walk within the hospital room or to the bathroom. Severely restricted mobility was defined as being confined to the bed or to a chair at the bedside. 


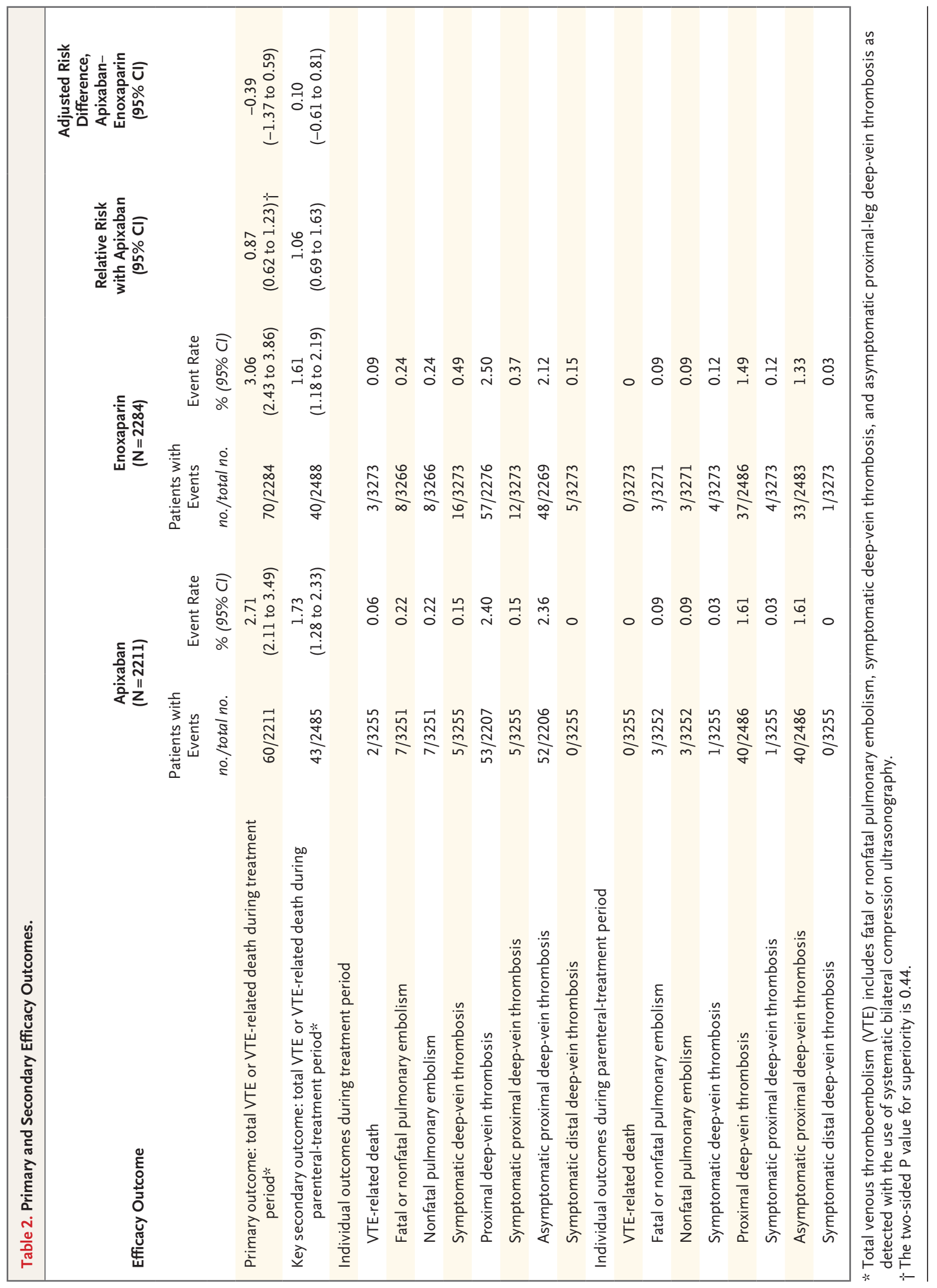

N ENGLJ MED 365;23 NEJM.ORG DECEMBER 8, 2011 


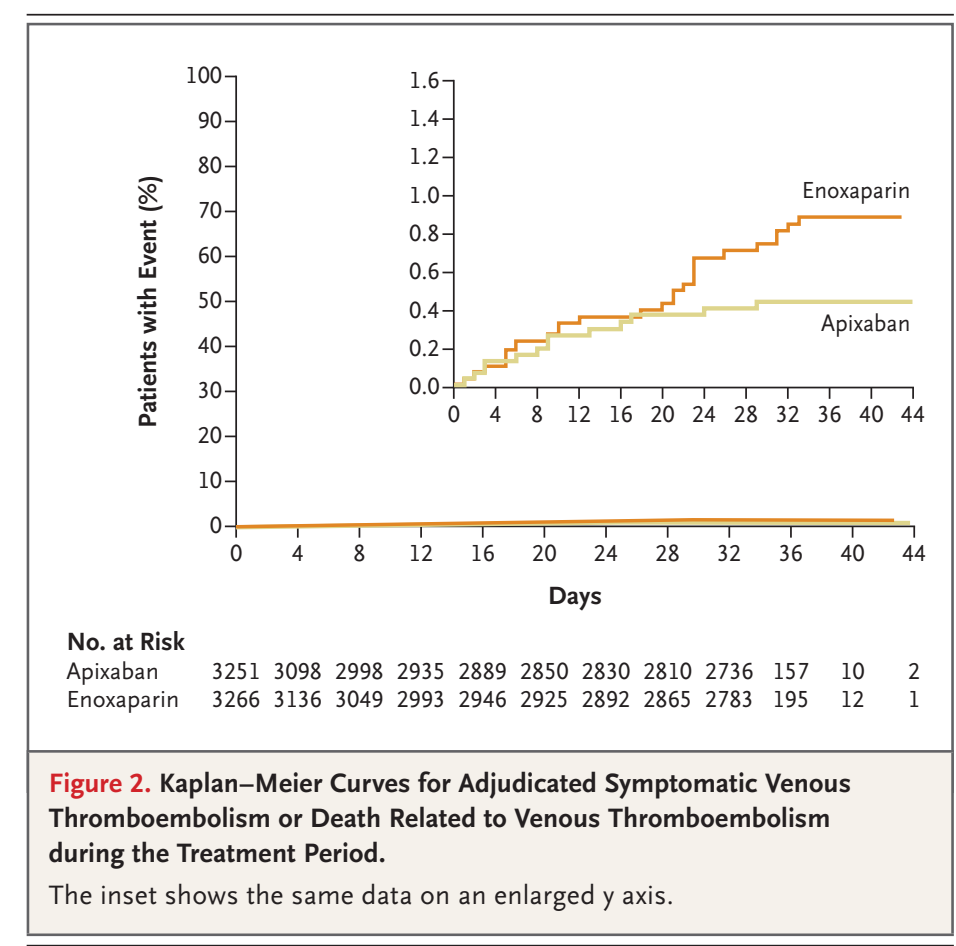

secondary efficacy outcome of the composite of total venous thromboembolism (i.e., asymptomatic proximal deep-vein thrombosis, proximal or distal symptomatic deep-vein thrombosis, or pulmonary embolism) or death related to venous thromboembolism evaluated at the end of the parenteraltreatment period occurred in $1.73 \%$ of patients in the apixaban group (43 of 2485 patients) and in $1.61 \%$ in the enoxaparin group (40 of 2488 patients) (relative risk, 1.06 ; $95 \% \mathrm{CI}, 0.69$ to 1.63 ). The rates of the primary and secondary efficacy outcomes are shown in Table 2. The results of the primary efficacy outcome were consistent across all prespecified subgroups.

The rate of symptomatic deep-vein thrombosis was lower among patients who received extended thromboprophylaxis with apixaban than among those who received enoxaparin $(0.15 \%$ [ 5 of 3255 patients] vs. $0.49 \%$ [ 16 of 3273 patients]), but this difference did not reach significance. The cumulative rates of any symptomatic venous thromboembolism, including death related to venous thromboembolism, are shown in Figure 2.

\section{SAFETY OUTCOME}

Table 3 shows the results of the bleeding outcomes. Major bleeding events during the 30-day treatment period occurred in $0.47 \%$ of the patients in the apixaban group (15 of the 3184 patients who received at least one dose of apixaban) and in $0.19 \%$ in the enoxaparin group ( 6 of the $3217 \mathrm{pa}-$ tients who received at least one dose of enoxaparin) (relative risk with apixaban, 2.58; 95\% CI, 1.02 to $7.24 ; \mathrm{P}=0.04)$. Major plus clinically relevant nonmajor bleeding occurred in $2.67 \%$ of the patients who received apixaban (85 of 3184) and in 2.08\% of those who received enoxaparin (67 of 3217) (relative risk, 1.28 ; $95 \% \mathrm{CI}, 0.93$ to $1.76 ; \mathrm{P}=0.12$ ). The rates of total bleeding events in the apixaban and enoxaparin groups were $7.73 \%$ (246 of 3184) and $6.81 \%$ (219 of 3217), respectively (relative risk, 1.13 ; $95 \% \mathrm{CI}, 0.95$ to $1.34 ; \mathrm{P}=0.18$ ). Figure 3 shows the Kaplan-Meier curves for major and clinically relevant nonmajor bleeding in both groups.

There was no significant difference in the rate of death between the apixaban group and the enoxaparin group $(4.1 \%$ in each group [131 and 133 patients, respectively]). The rates of adverse events, including myocardial infarction, stroke, and thrombocytopenia, did not differ significantly between the two groups during the treatment period or the follow-up period. The rates of elevation of the alanine aminotransferase and total bilirubin levels to at least 3 times the upper limit of the normal range did not differ between the two groups.

\section{DISCUSSION}

The primary efficacy outcome, a composite of venous thromboembolism and death related to venous thromboembolism, occurred in $2.71 \%$ of the patients randomly assigned to apixaban and in $3.06 \%$ of those assigned to enoxaparin $(\mathrm{P}=0.44)$. However, there was an almost immediate increase in the risk of events when enoxaparin was stopped. After the parenteral-treatment period, the primary efficacy outcome occurred in $31 \mathrm{pa}$ tients in the enoxaparin group but in only $18 \mathrm{pa}-$ tients receiving extended treatment with apixaban (relative risk with apixaban, 0.59\%; 95\% CI, 0.33 to 1.05 ). When only symptomatic venous thromboembolism and death related to venous thromboembolism were included, extended treatment with apixaban reduced events from 18 to 8 (relative risk, $0.44 ; 95 \%$ CI, 0.19 to 1.00 ). Therefore, even though our trial was negative, the strategy of extended prophylaxis with apixaban may have promise.

The ADOPT trial was underpowered. The $13 \%$ reduction in the primary outcome favored apixaban, but the between-group difference was not significant, and thus no clinically directive 


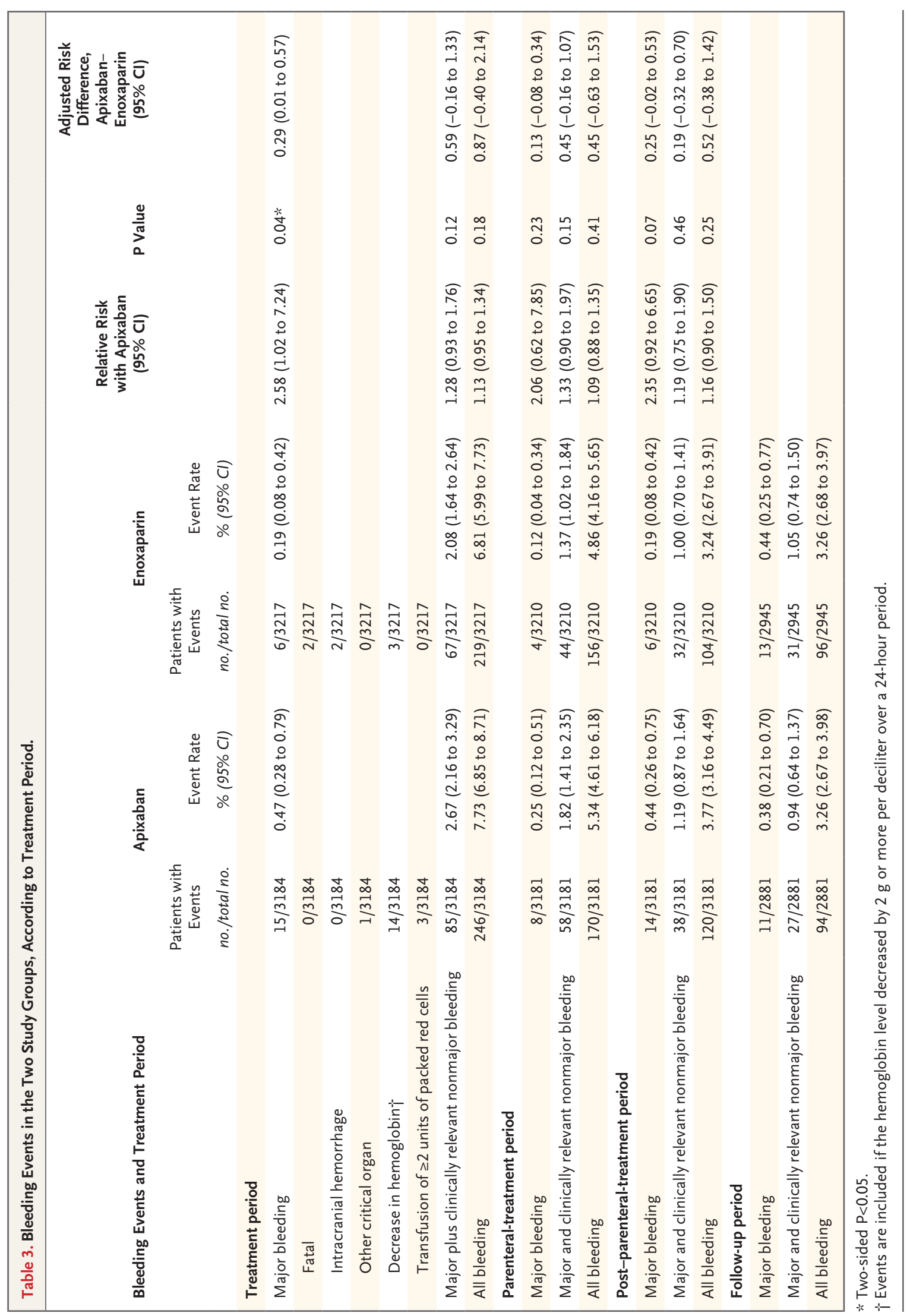

N ENGLJ MED 365;23 NejM.ORG DECEMBER 8, 2011

The New England Journal of Medicine 


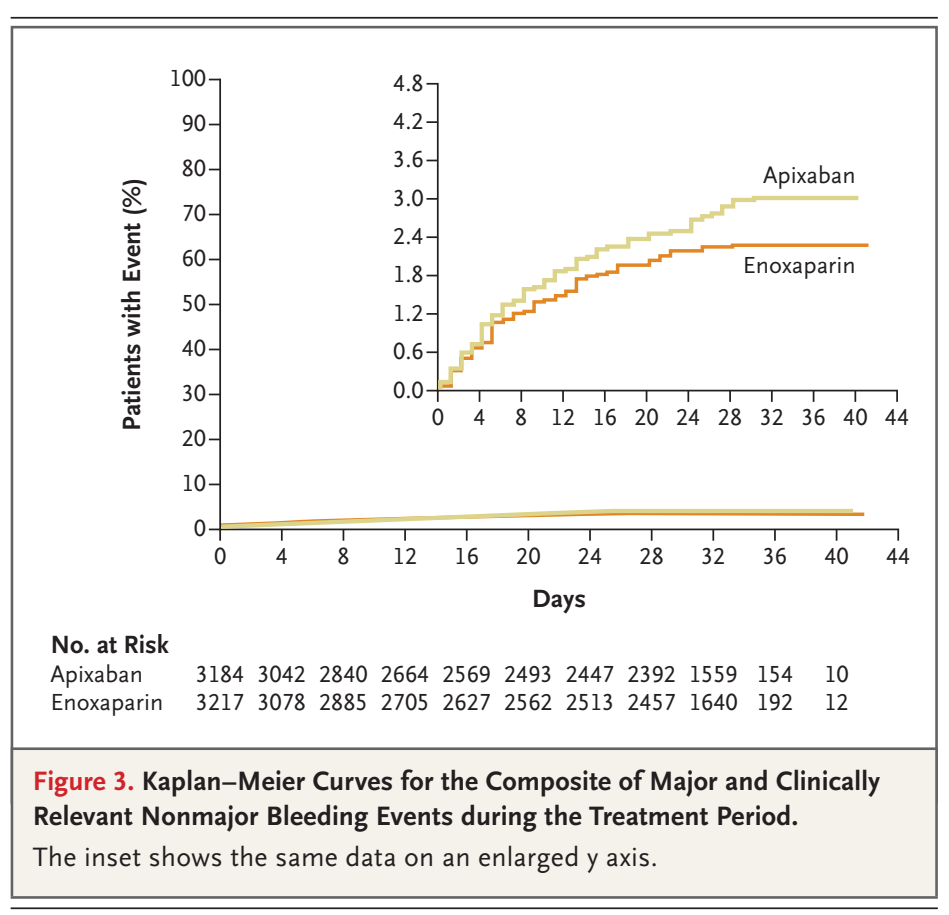

conclusion can be drawn. The results of this trial may not be applicable to typical populations of hospitalized medically ill patients because screening for venous thromboembolism with the use of compression ultrasonography is not performed routinely at the time of hospital discharge. Furthermore, the curves between apixaban and enoxaparin began to separate well after the final dose of enoxaparin was administered, suggesting that the study outcome might have been positive if we had extended the duration of apixaban therapy for more than 30 days.

The comparator in this trial was enoxaparin administered for 6 to 14 days. Although this is the licensed regimen for enoxaparin prophylaxis in medically ill patients, most patients who are hospitalized for a medical illness remain in the hospital for fewer than 5 days. It is standard practice to stop enoxaparin at the time of discharge, even in patients with persistent risk factors for venous thromboembolism. Thus, the design of this trial favored better efficacy in the enoxaparin group than would be expected with ordinary clinical care because patients in the enoxaparin group received prophylaxis for a longer duration than usual.

The results of the ADOPT trial warrant comparison with two other contemporary trials evaluating extended thromboprophylaxis in medically ill patients. In the EXCLAIM trial, ${ }^{8} 5963$ medically ill patients were given open-label enoxaparin $(40 \mathrm{mg}$ once daily) for an average of 10 days. As compared with placebo, extended prophylaxis with enoxaparin reduced the rate of venous thromboembolism from $4.0 \%$ to $2.5 \%$ but increased the rate of major bleeding from $0.3 \%$ to $0.8 \%$.

The MAGELLAN trial compared extended prophylaxis with oral rivaroxaban (10 mg once daily) with a 6-to-14-day course of subcutaneous enoxaparin (40 mg once daily) in 8101 medically ill patients. ${ }^{9}$ At day 35, the rate of the primary efficacy outcome was significantly lower with extended rivaroxaban than with enoxaparin $(4.4 \%$ vs. $5.7 \%$; hazard ratio with rivaroxaban, $0.77 ; 95 \% \mathrm{CI}, 0.62$ to $0.96 ; \mathrm{P}=0.02$ ). However, there was an increase in treatment-related major and clinically relevant nonmajor bleeding with rivaroxaban as compared with enoxaparin both during the period from 1 to 10 days ( $2.8 \%$ and $1.2 \%$, respectively) and during the period from 11 to 35 days $(1.4 \%$ and $0.5 \%$, respectively).

In the ADOPT trial, the rate of major bleeding with apixaban was less than $0.5 \%$. This rate is consistent with the safety of the regimen of $2.5 \mathrm{mg}$ of apixaban twice daily, which is the regimen that is licensed in Europe for thromboprophylaxis after elective hip- or knee-replacement surgery.

The strengths of the current trial include the large sample size; the randomized, double-blind, double-dummy design; the centralized adjudication of all suspected outcomes by a committee whose members were unaware of the treatment assignments; and the enrollment of a broad population of medically ill patients. Some limitations are the inclusion of asymptomatic proximal deep-vein thrombosis as part of the primary efficacy outcome and the fact that a follow-up ultrasound examination was performed in only $64 \%$ of the patients, which reduced the statistical power of the trial. Compression ultrasonography is not performed routinely in medical patients at the time of discharge from the hospital, and is performed even more rarely at 1 month after discharge. The low rate of asymptomatic proximal deep-vein thrombosis among patients who received short-term prophylaxis validates this approach. In the ADOPT and MAGELLAN trials, the logistic complexity of performing compression ultrasonography in this frail patient population before discharge from the hospital and at 30 days 
after discharge probably explains the suboptimal rates of follow-up examinations.

The ADOPT trial does not provide evidence to justify a policy of extended prophylaxis in a broad population of medically ill patients after hospital discharge. However, with event rates of venous thromboembolism at 30 days that range from 3\% in the ADOPT trial to $5 \%$ and $6 \%$ in the EXCLAIM and MAGELLAN trials, respectively, it is clear that the risk of venous thromboembolism increases beyond the time of hospital discharge. More precise risk-stratification methods are needed to identify a narrower spectrum of medically ill patients who may benefit from extended prophylaxis.

Supported by Bristol-Myers Squibb and Pfizer.

Dr. Goldhaber reports receiving consulting fees from Boehringer Ingelheim, Bristol-Myers Squibb, Daiichi Sankyo, Eisai, EKOS, Medscape, Merck, Portola, and Sanofi-Aventis and grant support through his institution from Boehringer Ingelheim, Bristol-Myers Squibb, Eisai, EKOS, Johnson \& Johnson, and Sanofi-Aventis; Dr. Leizorovicz, holding board memberships at Bayer, GlaxoSmithKline, Leo Pharma, and Sanofi-Aventis and receiving consulting fees from Bayer, Boehringer Ingelheim, and GlaxoSmithKline, grant funding through his institution from GlaxoSmithKline, lecture fees from Leo Pharma, and payment for development of educational presentations from Bayer; Dr. Kakkar, receiving consulting fees from Bayer Healthcare, Boehringer Ingelheim, Bristol-Myers Squibb, Daiichi Sankyo, Eisai, GlaxoSmithKline, Pfizer, and Sanofi-Aventis, grant support through his institution from Bayer Healthcare, Boehringer Ingelheim, Eisai, Pfizer, and Sanofi-Aventis, and lecture fees from Bayer Healthcare, Boehringer Ingelheim, Bristol-Myers Squibb, Eisai, GlaxoSmithKline, Pfizer, and Sanofi-Aventis; Dr. Haas, receiving consulting fees from Bayer, Boehringer Ingelheim, Bristol-Myers Squibb, Daiichi Sankyo, Isis, and SanofiAventis, lecture fees from Bayer and Boehringer Ingelheim, and payment for the development of educational presentations from Bayer and Bristol-Myers Squibb; Dr. Merli, receiving consulting fees and grant funding through his institution from Bayer and Sanofi-Aventis; Dr. Knabb, being an employee of Bristol-Myers Squibb and receiving stock or stock options as part of his compensation as an employee; and Dr. Weitz, receiving consulting fees from Bayer, Boehringer Ingelheim, Bristol-Myers Squibb, Daiichi Sankyo, Johnson \& Johnson, and Pfizer. No other potential conflict of interest relevant to this article was reported.

Disclosure forms provided by the authors are available with the full text of this article at NEJM.org.

We thank Kathryn Mikkelsen for assistance with the preparation of the manuscript; and Dalei Chen, Ph.D., and Yun Peng, Ph.D., for statistical support.
REFERENCES

1. Goldhaber SZ, Visani L, De Rosa M. Acute pulmonary embolism: clinical outcomes in the International Cooperative Pulmonary Embolism Registry (ICOPER). Lancet 1999;353:1386-9.

2. Collins R, Scrimgeour A, Yusuf $S$, Peto R. Reduction in fatal pulmonary embolism and venous thrombosis by perioperative administration of subcutaneous heparin: overview of results of randomized trials in general, orthopedic, and urologic surgery. N Engl J Med 1988;318:1162-73.

3. Samama MM, Cohen AT, Darmon JY, et al. A comparison of enoxaparin with placebo for the prevention of venous thromboembolism in acutely ill medica patients. N Engl J Med 1999;341:793-800 4. Leizorovicz A, Cohen AT, Turpie AG, Olsson CG, Vaitkus PT, Goldhaber SZ. Randomized, placebo-controlled trial of dalteparin for the prevention of venous thromboembolism in acutely ill medical patients. Circulation 2004;110:874-9.

5. Cohen AT, Davidson BL, Gallus AS, et al. Efficacy and safety of fondaparinux for the prevention of venous thromboembolism in older acute medical patients: randomised placebo controlled trial. BMJ 2006;332:325-9. 6. Kakkar AK, Brenner B, Dahl OE, et al. Extended duration rivaroxaban versus shortterm enoxaparin for the prevention of venous thromboembolism after total hip arthroplasty: a double-blind, randomised controlled trial. Lancet 2008;372:31-9.

7. Geerts WH, Bergqvist D, Pineo GF, et al. Prevention of venous thromboembolism: American College of Chest Physicians Evidence-Based Clinical Practice Guidelines (8th Edition). Chest 2008;133:381S-453S. 8. Hull RD, Schellong SM, Tapson VF, et al. Extended-duration venous thromboembolism prophylaxis in acutely ill medical patients with recently reduced mobility: a randomized trial. Ann Intern Med 2010;153:8-18.

9. Rivaroxaban compares favorably with enoxaparin in preventing venous thromboembolism in acutely ill patients without showing a net clinical benefit. Washington, DC: American College of Cardiology, April 5, 2011 (http://www.cardiosource.org/ News-Media/Media-Center/News-Releases/ 2011/04/MAGELLAN.aspx).

10. Lassen MR, Gallus A, Raskob GE, Pineo G, Chen D, Ramirez LM. Apixaban versus enoxaparin for thromboprophylaxis after hip replacement. N Engl J Med 2010;363:2487-98.

11. Lassen MR, Raskob GE, Gallus A, Pineo G, Chen D, Hornick P. Apixaban versus enoxaparin for thromboprophylaxis after knee replacement (ADVANCE-2): a randomised double-blind trial. Lancet 2010; 375:807-15.

12. Connolly SJ, Eikelboom J, Joyner C, et al. Apixaban in patients with atrial fibrillation. N Engl J Med 2011;364:806-17.

13. Granger CB, Alexander JH, McMurray $\mathrm{JJ}$, et al. Apixaban versus warfarin in patients with atrial fibrillation. N Engl J Med 2011;365:981-92.

Copyright (C) 2011 Massachusetts Medical Society. 